\title{
EDUCATION
}

\section{Pride and Prejudice and Learning: An Interprofessional Experience with CLS and Nursing Students}

\author{
KRISTINA JACKSON BEHAN, KRISTEN COFFEY, MICHELE PROMO, \\ TERESA BROOKS, JILL VAN DER LIKE
}

\begin{abstract}
Literature is scarce regarding medical laboratorians and their attitudes about interprofessional interactions with other healthcare providers. We investigated learning and attitudes in a joint project that brought Clinical Laboratory Sciences (CLS) students and Nursing students together. The nursing and CLS faculty created a simulated post-partum patient who developed deep vein thrombosis followed by pulmonary embolism. The patient was heterozygous for the Factor $\mathrm{V}$ Leiden mutation. The simulations occurred in two venues. The patient scenario occurred at the student Nursing Skills and Simulation Learning Center "SIM lab" at the bedside of the patient experiencing symptoms of deep vein thrombosis and pulmonary embolism, with the nursing students responding to the patient's distress. CLS students collected blood from the patient during the crisis. The laboratory scenario occurred in the CLS teaching laboratory. CLS students performed real time PCR on the patient for the Factor V Leiden mutation, and instructed the nursing students how to interpret the results.
\end{abstract}

Learning gains were measured by survey after the 2 events. Retention of learning was measured 6 weeks after the second event took place. All students showed sustained learning about venous thromboembolism, its risk factors, and genetic mutations that predispose towards thrombophilia.

Students' attitudes about interprofessional education and each other's professions were surveyed before and after the experience. Students valued the experience and $87 \%$ of them responded that they are interested in pursuing more interprofessional education training opportunities.

ABBREVIATIONS: IPE - Interprofessional Education, VTE - Venous Thromboembolism, DVT - Deep Vein
Thrombosis, PE - Pulmonary Embolism. NSSL Nursing Student Simulation Lab, CLS-Clinical Laboratory Sciences

INDEX TERMS: Interprofessional Relations, Hand Hygiene, Simulation Training, Patient Identification Systems

Clin Lab Sci 2017;30(4):233

Kristina Jackson Behan, PhD, MLS (ASCP), University of West Florida. College of Health, Pensacola, FL

Kristen Coffey, MS, MB (ASCP), University of West Florida. College of Health, Pensacola, FL

Michele Promo, PhD, MLS (ASCP), University of West Florida. College of Health, Pensacola, FL

Teresa Brooks, MLS (ASCP), University of West Florida. College of Health, Pensacola, FL

Jill Van Der Like, DNP, MSN, RNC, University of West Florida, College of Health, Pensacola, FL

Address for Correspondence: Kristina Behan, $P h D, M L S$ (ASCP), University of West Florida, Bldg. 58, Room 81, 11000 University Parkway, Pensacola, FL 32514, 850474-3060,kbehan@uwf.edu

\section{INTRODUCTION}

The competency domains of interprofessional education (IPE) include teamwork, communication, roles (hierarchy and professional pride) and values (respect and prejudice). ${ }^{1}$ The literature has many reports of nursing students involved in IPE. There are far fewer studies that specifically look at IPE involving Clinical Laboratory Sciences (CLS) students and nursing students together. 


\section{EDUCATION}

In fact, the Interprofessional Education Collaborative Expert Panel, which is sponsored by AACN (Nursing), AACOM (Osteopathic Medicine), ASPH (Public Health), AACP (Pharmacy), ADEA (Dentistry) and AAMC (Medical Colleges), does not have representatives from medical laboratories. Without best practice models to refer to, CLS programs that develop IPE programs must do so by experimentation and creative solutions. ${ }^{2}$ The IPE literature recommends that projects should appeal to all students involved and the content should be relevant to their professions. ${ }^{3}$

We created an IPE project that brought CLS and nursing students together, and we investigated their learning gains. We also investigated their professional pride and prejudices. Students worked in small groups on a simulated patient with venous thromboembolism (VTE), beginning with symptoms of deep vein thrombosis (DVT) and progressing to pulmonary embolism (PE). We chose VTE for the project because it is a medical condition that requires both nursing skills in observation and reaction, and high complexity laboratory testing to diagnose. As such, the simulations appealed to both sets of students and posed an opportunity for deliberate practice of their skills.

VTE is a major cause of hospital related deaths and the leading cause of maternal death in the US. ${ }^{4,5}$ Risk factors identified in postpartum women include being bedridden, hypertension, heart disease, diabetes mellitus, obesity, race, age, and smoking. Symptoms of DVT and $\mathrm{PE}$ include heat, edema in the leg, decrease in peripheral pulse, drop in oxygen saturation and loss of consciousness. ${ }^{6}$ It is possible to simulate these symptoms with a Laerdal (Wappingers Falls, NY) manikin "patient".

Thrombophilia has the greatest influence on the development of VTE in pregnant and post-partum women, with an odds ratio of 51.8. ${ }^{4}$ Factor V Leiden and Prothrombin G20210A mutations are the most commonly identified inherited causes of thrombophilia. ${ }^{5}$ These mutations can be identified by real time PCR. ${ }^{7}$

The VTE patient scenario provided CLS and nursing students a realistic learning experience that also promoted IPE communication and respect for the others' professional roles. The project occurred in two venues, first at the bedside of the manikin patient experiencing symptoms of DVT and PE in the nursing student simulation lab (NSSL, also known as the SIM-lab), and second in the CLS teaching laboratory performing DNA analysis on the patient. Student learning gains and attitudes were measured by survey.

\section{METHODS}

The faculty from the Nursing Department and Clinical Laboratory Sciences Program identified two student groups based on their availability to participate in both events. Eighty-nine students in total participated in the experience. The nursing students were seniors earning a Bachelor of Science in Nursing, and had started their clinical experience. The CLS students were juniors earning a Bachelor of Science in Clinical Laboratory Sciences, and taking the course Molecular Diagnostics. All of the students had prior experience with SIM-lab. The project was approved by the Institutional Review Board at the University of West Florida.

All of the students were acquainted with VTE from their respective courses. Students were surveyed a total of 3 times using Qualtrics survey software (Qualtrics, LLC). Statistical analysis was performed using SAS statistical software. Students were surveyed prior to the first event for their knowledge about DVT and PE, and their attitudes about interprofessional training and teamwork. They were surveyed again immediately after the second event. These are referred to as Survey \#1 and Survey \#2. Six weeks after the exercise, students were surveyed a third time. This is referred to as Survey \#3. A one-way repeated measured analysis of variance (ANOVA) was conducted to evaluate the null hypothesis that there was no change in participants' scores on identifying risk factors when measured before, during, and 6-weeks after participation in education.

Students were divided into small groups for each of the SIMs. There were 6 groups of students that participated in the nursing student SIM-lab. There were 8 groups of students that participated in the CLS lab. Each group was about 8-15 students, and contained both CLS and nursing students.

\section{The NSSL SIM- lab patient experience}

The NSSL event was 2 hours long. Acting roles were assigned to the nursing students as the primary, secondary and resource nurses and the patient's partner. The role of the physician was played by the nursing 
faculty by phone. Two CLS student actors were assigned to collect a blood sample when called. The patient Jenny Jones was a Laerdal manikin. Figure 1 shows the student actors during the SIM.

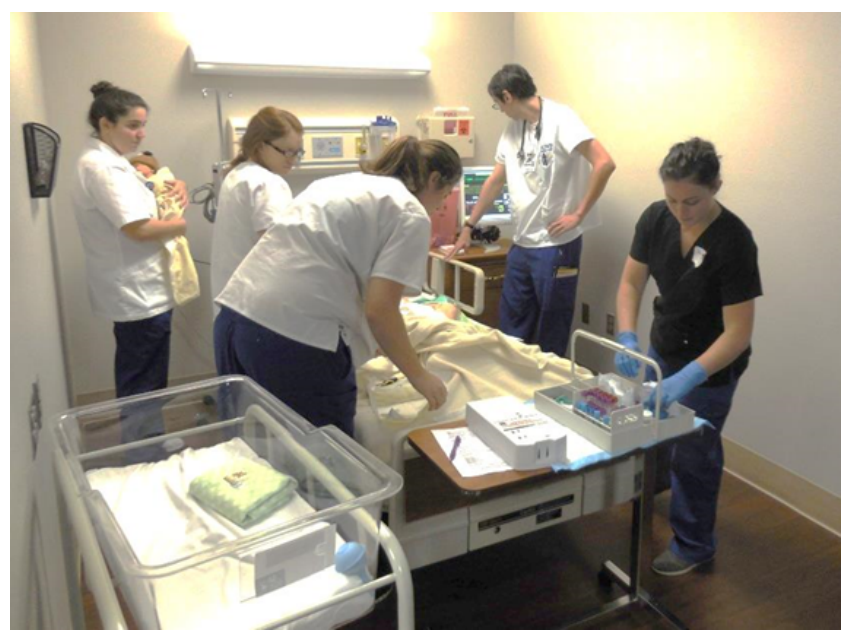

Figure 1. Students respond to patient Jenny Jones during the nursing SIM lab event

Relevant patient history and physical findings were provided on an RN report worksheet during a pre-event briefing. The patient Jenny Jones was 5 days post-partum after a C-section. She was reluctant to ambulate, but alert and oriented. Her laboratory results were a hemoglobin of $9 \mathrm{~g} / \mathrm{dL}$ and hematocrit $28 \%$. She was on room air. During the simulation, Jenny Jones complained of shortness of breath and pain in the calf. Her oxygen saturation dropped, and right lung sounds were absent. She became disoriented and then lost consciousness. The physician ordered D-dimer, prothrombin time, activated partial thromboplastin time, arterial blood gases and genetic testing stat. The CLS students collected blood while the nursing students responded to the patient's distress. The simulation was video recorded. Students who were not actors observed the activity remotely in the debriefing room. Following the SIM, the actors' responses were analyzed by all students during a debriefing session.

\section{The CLS lab experience (two weeks later)}

Both CLS and nursing students were assigned to each working group. Before this event, CLS students designed an educational PowerPoint (PPT) to present to their group's nursing students. PPT content included the role of Factor $\mathrm{V}$ in coagulation, the mutation Factor V Leiden and how it leads to thrombophilia by activated Protein C resistance, and real time PCR methodology.
During this laboratory experience, the CLS students performed a real time PCR (Step One, Life Technologies) analysis for Factor $\mathrm{V}$ Leiden on simulated heterozygous patient DNA, using a modified procedure by Luderer et al. ${ }^{7}$ Each student group set up appropriate controls, and explained the role of each control to the nursing students (Figure 2). The real time PCR results were available in 90 minutes, and each control was interpreted by the CLS student. The nursing students were asked to interpret the results for the patient Jenny Jones, and their interpretation was either confirmed or further discussed by the CLS students.

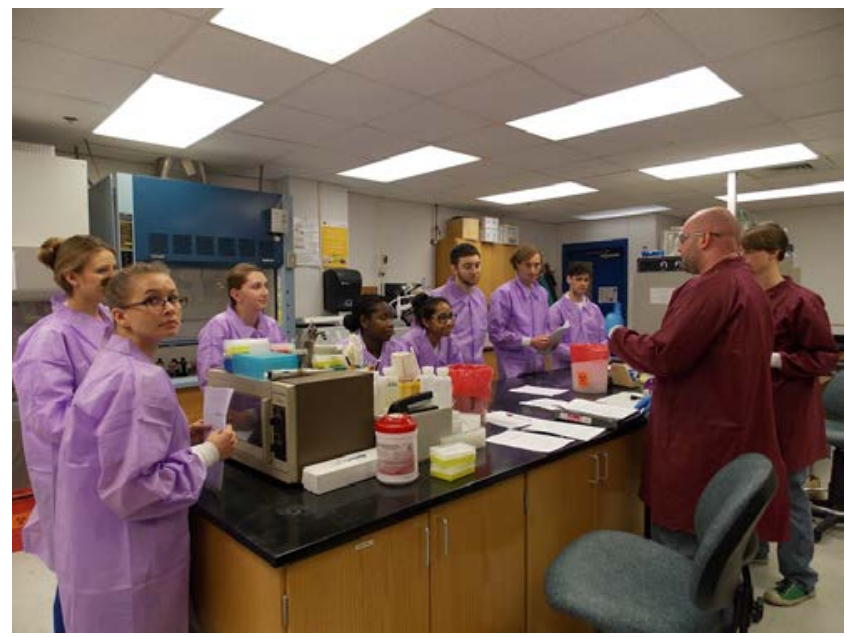

Figure 2. CLS students engage nursing students during Factor V Leiden real time analysis

At the conclusion of the laboratory event, there was a reception with refreshments. This phase provided a time for Survey \#2 to be completed and an increased opportunity for interprofessional communication. Students were incentivized to complete the survey with gift cards for coffee.

Six weeks after the CLS lab event, Survey \#3 was emailed to all participants. This survey followed a holiday break, during which time the students were not further exposed to the subject matter.

\section{RESULTS}

Students were assessed for their pre-event and post-event knowledge about the clinical features and risks of DVT and PE. Although all of the students were surveyed, not all of them completed each survey. Sixty-six students completed both Survey \#1 and \#2 (56\% nursing and $44 \%$ CLS students). Six weeks later, only 38 students 
(54\% nursing and 46\% CLS students) completed Survey \#3. In all, 29 of the original 89 participants replied to each of the 3 surveys.

Table 1 shows results from the students who completed both Survey \#1 and \#2. The table shows the percent of students who correctly identified each of the clinical features and risk factors listed. Table 1 also shows the results of all of the students who completed Survey \#3. ANOVA was conducted to evaluate the null hypothesis that there was no change in participants' scores on identifying risk factors when measured before, during, and 6-weeks after participation in education. The results indicated a significance time effect, Wilks' Lambda $=.14$, $\mathrm{F}(2,7), \mathrm{p}<.001$ and $\eta 2=.861$. Thus there was significant evidence to reject the null hypothesis. Follow up comparisons indicated that each pairwise difference was significant, with a p-value less than .01 . There was a significant increase over time in the number of students who correctly identified the risk factors and symptoms of VTE, suggesting that participation in the course increased the participants' level of knowledge.

The survey also focused on attitudes regarding interprofessional respect and training. This evaluates learning in the affective domain. Statements were slanted to uncover professional pride and prejudice, and students either agreed, were neutral, or disagreed with these statements. Table 2 summarizes the results. Ten percent of CLS students agreed with the statement "Nursing staff contributes more to patient outcomes than laboratory staff' before the events; only 3\% agreed afterwards. Forty one percent of nursing students agreed with the statement before the events, but only $14 \%$ of them agreed afterwards.

Before the events, 97\% of the CLS students disagreed with or were neutral towards the statement "The academic training for nurses is more rigorous that the training for clinical laboratory sciences". They held fast to that opinion after the events, but many nursing students changed their opinion. Before the events only $24 \%$ of nursing students disagreed with the statement. After the events, $49 \%$ of nursing students disagreed.

None of the nursing students agreed with the statement "Laboratory testing for genetic mutations is easy to perform and interpret" before the event, and 5\% agreed with it afterwards. More CLS students changed their attitudes about the statement. Before the event, 33\% of the CLS students disagreed with the statement. After the events 55\% disagreed with the statement.

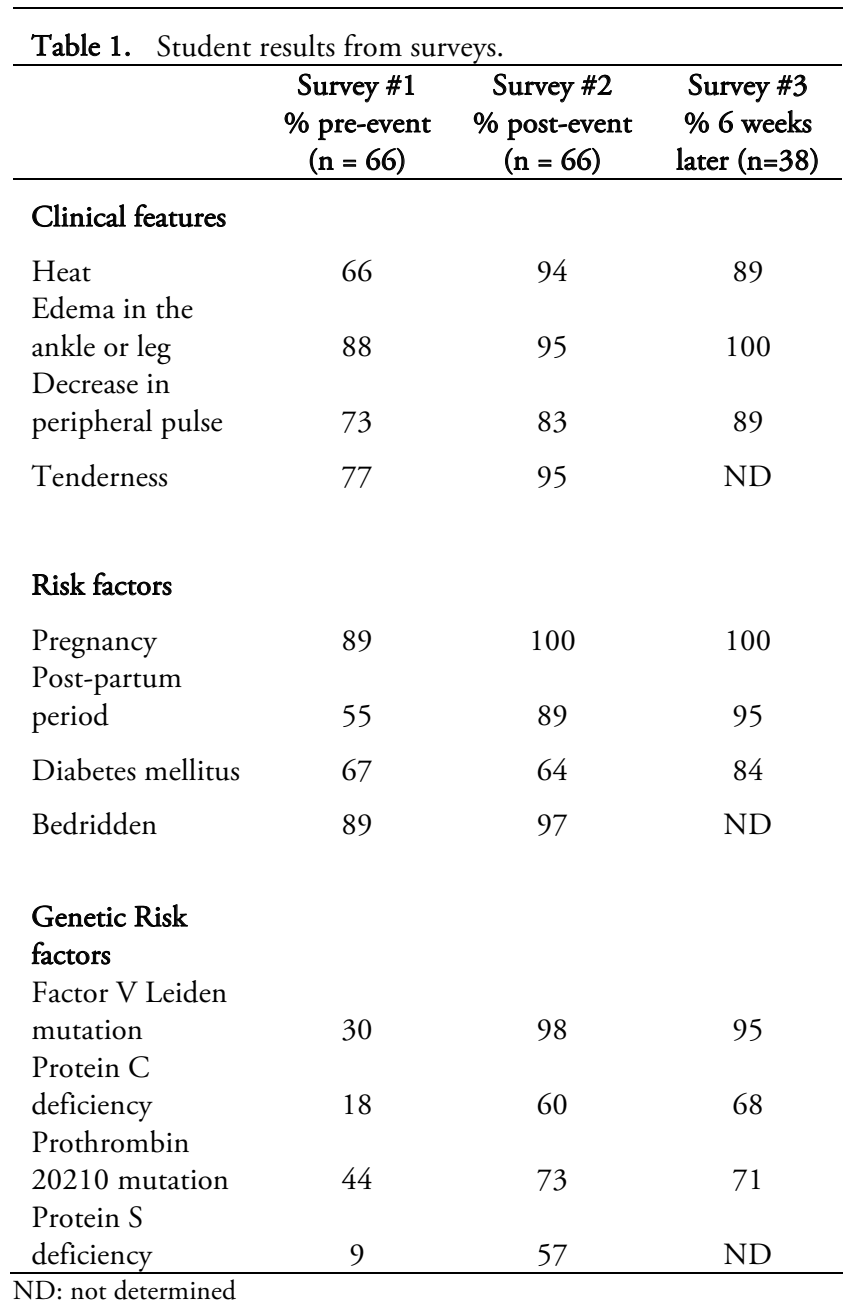

Students were queried about their attitudes towards interprofessional education 6 weeks after the events by Survey \#3. Figure 3 summarizes the value that the students placed on interprofessional communication and training 6 weeks after the completion of the project, showing growth in the affective domain.

We saw a gain in the students' perception of how each profession contributes to diagnosis by listing different medical professions and asking the question "Is this provider involved in the diagnosis of DVT and PE?". Prior to the events, $86 \%$ of all the students felt that nurses were involved, and $80 \%$ felt that CLS/MLS were involved in diagnosis. After the events $94 \%$ of the students felt that nurses are involved in the diagnosis and 97\% felt that CLS/MLS are involved. 
Table 2. Results of survey on attitudes regarding interprofessional respect and training.

\begin{tabular}{lccc}
\hline $\begin{array}{l}\text { Nursing staff contributes more } \\
\text { to patient outcomes than } \\
\text { laboratory staff }\end{array}$ & $\begin{array}{c}\text { Disagree } \\
(\%)\end{array}$ & $\begin{array}{c}\text { Neutral } \\
(\%)\end{array}$ & $\begin{array}{c}\text { Agree } \\
(\%)\end{array}$ \\
\hline CLS students pre-event $(\mathrm{n}=29)$ & $52 \%$ & $41 \%$ & $10 \%$ \\
CLS students post-event & $55 \%$ & $41 \%$ & $3 \%$ \\
$\begin{array}{l}\text { Nursing students pre-event }(\mathrm{n}= \\
\text { 37) }\end{array}$ & $27 \%$ & $30 \%$ & $41 \%$ \\
Nursing students post-event & $51 \%$ & $35 \%$ & $14 \%$
\end{tabular}

\begin{tabular}{llll}
\hline $\begin{array}{l}\text { The academic training for nurses } \\
\text { is more rigorous than the } \\
\text { training for CLS/MLS }\end{array}$ & & & \\
\hline CLS students pre-event & $76 \%$ & $24 \%$ & $3 \%$ \\
CLS students post-event & $69 \%$ & $31 \%$ & $0 \%$ \\
Nursing students pre-event & $24 \%$ & $51 \%$ & $22 \%$ \\
Nursing students post-event & $49 \%$ & $35 \%$ & $16 \%$
\end{tabular}

\begin{tabular}{lccc}
\hline $\begin{array}{l}\text { Laboratory testing for genetic } \\
\text { mutations is easy to perform and } \\
\text { interpret }\end{array}$ & & & \\
\hline CLS students pre-event & $33 \%$ & $53 \%$ & $13 \%$ \\
CLS students post-event & $55 \%$ & $17 \%$ & $28 \%$ \\
Nursing students pre-event & $62 \%$ & $32 \%$ & $0 \%$ \\
Nursing students post-event & $68 \%$ & $19 \%$ & $5 \%$ \\
\hline
\end{tabular}

\section{DISCUSSION}

Following the two-part project, the participants showed significant and sustained gains in their knowledge about venous thromboembolism (VTE). Clinical features were correctly identified by more students. Many students already held knowledge about risk factors, such as pregnancy and being bed-ridden before the project, and more students were able to identify these risks after the project. Interestingly, diabetes mellitus was only moderately recognized as a risk factor before and after the event. This is significant because diabetes was not part of the scenario: the patient Jenny Jones was not described as having diabetes. Six weeks after the event, however, more students correctly identified diabetes mellitus as a risk for VTE. One possible explanation is that the students were curious enough about VTE to do more extensive study. Another explanation may be that the students who completed Survey \#3 had also answered correctly in Survey \#2, as there were less students who completed Survey \#3.

The most profound cognitive learning gains after the events were in the identification of genetic risk factors for VTE. Most participants (98\%) identified the Factor V Leiden mutation as a genetic risk. Significantly, there were also sustained learning gains in the correct identification of Protein C deficiency, Protein S deficiency and Prothrombin G20210A, shown in Table 1. These risks for thrombophilia are related to activated protein $\mathrm{C}$ resistance, and were highlighted by the CLS students in their PowerPoint presentations. We saw a positive growth in students' affective domain. CLS students expressed their professional pride that laboratory staff contributes significantly to patient outcomes. Preconceived prejudice held by the nursing students lessened as they gained an appreciation for the

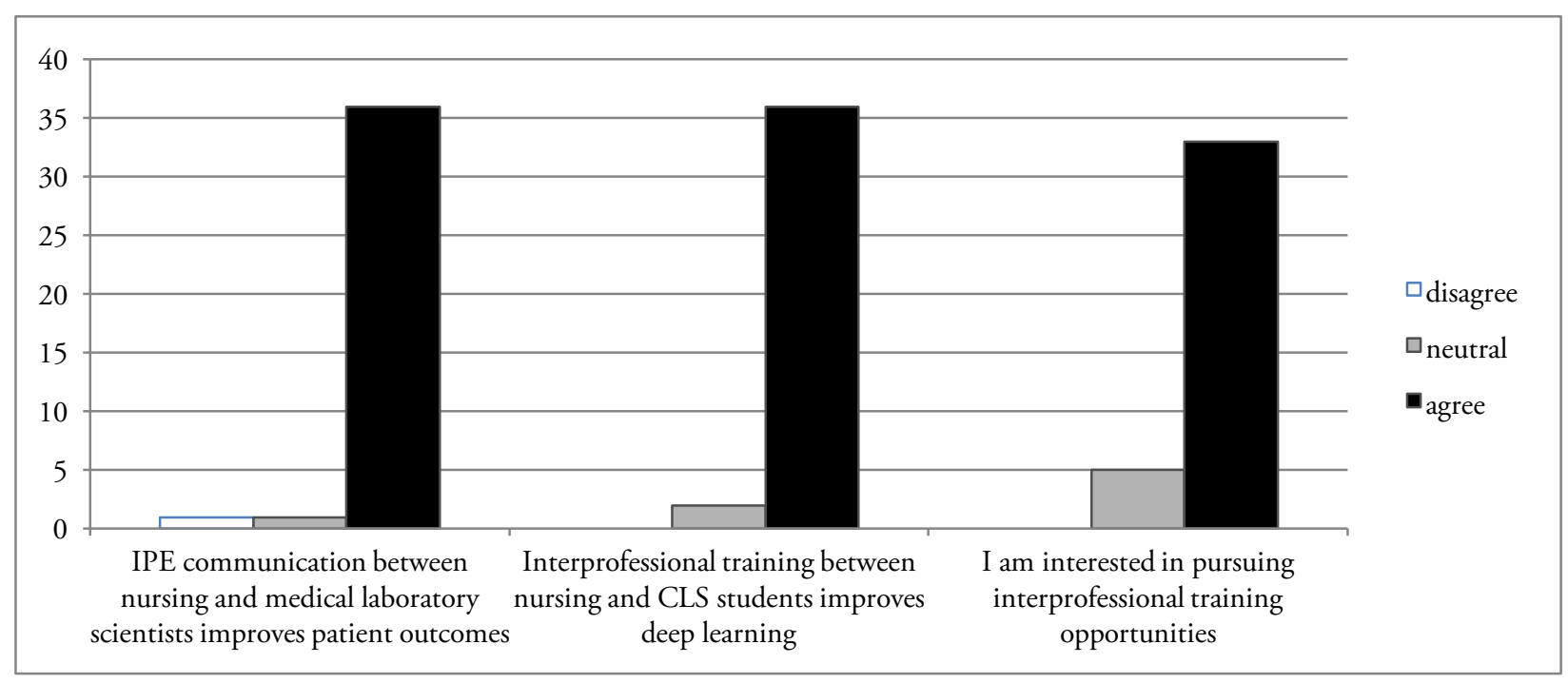

Figure 3. Professional pride. Six-week post events attitudes of all attendees regarding IPE. Number of students with given response. 


\section{EDUCATION}

laboratory's role in patient outcomes. Ultimately only $14 \%$ of the nursing students felt that nursing staff contributes more to patient outcomes than laboratory staff. After the two-part event almost half of the nursing students disagreed with a statement that their training was more rigorous than CLS training.

None of the nursing students agreed with the statement that laboratory testing for genetic mutations is easy to perform, and this did not change after the event. 33\% of the CLS students disagreed with this statement before the event, but $55 \%$ disagreed afterwards. This demonstrates a boost in their own professional pride. Both sets of students appreciated the IPE experience and the majority of them hope to participate in IPE in the future.

The limitations of the project were the mismatch between the educational standing of the participants, the size of the sample, and the inconsistency of survey participation. The project had scheduling constraints. Faculty and students had to be available when the lab facilities were available. The students who participated were in courses taught by the faculty most interested in IPE. They were available by virtue of their class time, and many of them were enthusiastic about participating fully. Although the CLS students had no clinical experience to match the nursing students, they had sufficient university laboratory experience to compensate for it. The inconsistency of survey participation was unexpected. Student participation was mandatory during their class time, but Survey \#1 and Survey \#3 were done on the students' own time. Participation in Survey \#1 was not incentivized, and only $74 \%$ of the students completed it. Survey \#2 was incentivized with gift cards and the students were provided time to complete it at the conclusion of the event. This resulted in 98\% participation.

Incentives were important to the faculty as well. The faculty had to invest a significant amount of additional time and effort to design and implement a teaching scenario that would be meaningful to both nursing and CLS students. This was incentivized by a departmental grant which supported the purchase of a real time PCR analyzer. Other researchers have noted the importance of incentives and buy-in. Graybeal et al. noted in their work that financial support from administrators was important for the success of IPE projects. Importantly, they also noted that IPE projects that have strong faculty and administrative buy-in can actually modify the vision of a department. $^{2}$

\section{CONCLUSION}

Interprofessional education between nursing and CLS students is an opportunity to improve communication and promote respectful professional relationships. The simulated medical scenario helped students to retain knowledge. The majority of students involved in this project were enthusiastic participants, and are interested in pursuing more interprofessional training experiences. Simulated IPE provides an avenue for enhancing cognitive, psychomotor and affective learning in a controlled environment.

The faculty involved in this project were not officially surveyed as part of the project. In all, eight faculty members from both departments attended, observed, and provided feedback on some or all of the events. Interdepartmental communication was improved and professional relationships were forged. The authors are eager to work together to create more IPE experiences. We hope that the experience has made a significant impression on the students, and continues to influence their professional pride and eliminate prejudice towards each other as working professionals.

\section{ACKNOWLEDGEMENTS}

Katie Cavnar provided critical feedback during development and implementation of the project. Michael A Johnston performed the statistical analysis. The project was paid for by a grant from the University of West Florida College of Health.

\section{REFERENCES}

1. Interprofessional Education Collaborative Expert Panel. (2011). Core competencies for interprofessional collaborative practice: Report of an expert panel. Washington, D.C.: Interprofessional Education Collaborative.

2. Graybeal C, Long R, Scalise-Smith D, Zeibig E. The art and science of interprofessional education. J Allied Health. 2010;39 Suppl 1:232-7.

3. Tullmann DF, Shilling AM, Goeke LH, Wright EB, Littlewood KE. Recreating simulation scenarios for interprofessional education: an example of educational interprofessional practice. J Interprof Care 2013;27:426-8.

4. James AH, Jamison MG, Brancazio LR, Myers ER. Venous thromboembolism during pregnancy and the postpartum period: incidence, risk factors and mortality. Am J Obstet Gynecol 2006;194(5):1311-5.

5. D’Uva M, Di Micco, Strina I, DePlacido G. Venous thromboembolism and pregnancy. J Blood Med 2010;1:9-12. 


\section{EDUCATION}

6. Wells PS, Anderson DR, Bormanis J, Guy F, Mitchell M, Gray $\mathrm{L}$, et al. Value of assessment of pretest probability of deep-vein thrombosis in clinical management. Lancet. 1997;350 (9094):1795-8.
7. Luderer R, Verheul, Kortlandt W. Rapid detection of the Factor $\mathrm{V}$ Leiden mutation by real-time PCR with TaqMan minor groove binder probes. Clin Chem 2004;50(4):787-8. 